assessment. Cerebral palsy and cognitive impairment were studied according to early onset sepsis (EOS) and late onset sepsis (LOS) after adjustment for potential confounding variables using multivariate logistic regression models.

Results In total, 139 (5\%) of the 2665 live births included had an EOS alone (without LOS associated), 752 (28\%) a LOS alone (without EOS associated) and 64 (2\%) EOS and LOS associated. At 5 years, the rate of cerebral palsy was 9\% (157/1769) and cognitive impairment $12 \%(177 / 1495)$. Compared with uninfected infants, cerebral palsy was increased in the group of EOS alone $(\mathrm{OR}=1.70$, $95 \% \mathrm{CI}: 0.84-3.45)$, in the group of LOS alone (OR $=1.71,95 \% \mathrm{CI}$ : 1.14-2.56), and this risk was increased further when EOS and LOS were associated (OR $=2.33,95 \% \mathrm{CI}: 1.02-5.33)$. There was no association between neonatal infection and cognitive impairment.

Conclusion Neonatal infections among very preterm infants are associated with an increased risk of cerebral palsy at 5 years of age, particularly when EOS and LOS are cumulative.

\section{MATERNAL FATTY ACIDS INTAKE DURING PREGNANCY AND LATER CHILD COGNITIVE DEVELOPMENT IN THE EDEN MOTHER-CHILD COHORT STUDY}

doi:10.1136/archdischild-2012-302724.0207

1,2JY Bernard, 1,2M de Agostini, 1,2 A Forhan, 3,4V Champion, ${ }^{3,4} \mathrm{M}$ Kaminski, 1,2MA Charles, 1,2B Heude, EDEN Mother-child Cohort Study Group. ' Center for Research in Epidemiology and Population Health, Inserm, Villejuif; ${ }^{2}$ Faculté de Médecine, Université Paris-Sud 11, Le Kremlin-Bicêtre; ${ }^{3} E$ pidemiological Research Unit on Perinatal Health and Women's and Children's Health, Inserm Unit 953; ' Université Pierre et Marie Curie, Université Paris 6, Paris, France

Background and aims Polyunsaturated Fatty Acids (PUFA) are needed for child brain development, especially $n$-3 PUFAs. Prenatal exposure depends on maternal lipids intake during pregnancy. We aimed to investigate associations between maternal PUFAs intake during pregnancy and later child cognitive development.

Methods In 1066 children of the EDEN mother-child cohort, we assessed cognitive development at 3 years with the Ages and Stages Questionnaire (ASQ, score between 0 and 300). Maternal lipids intake during pregnancy was evaluated after delivery, using a food frequency questionnaire and a food-composition table. We investigated associations between PUFAs intake and ASQ score using multiple linear regressions adjusted for centre, child's age, gender and gestational age, maternal tobacco and alcohol consumptions, parental education, siblings, caregivers and preschool attendance.

Results Mean ASQ score was 270.1 ( \pm 29.4$), n-6 / n-3$ ratio in food intake was $10.0( \pm 2.3)$ and total $n-3$ PUFAs intake was $0.47 \%( \pm 0.09)$ of total energy intake. In crude analyzes, ASQ score was positively associated with each three $n$-3 PUFAs ( $\alpha$-linolenic, eicosapentaenoic and docosapentaenoic acids) and negatively with linoleic acid and $n-6 / n-3$ ratio. After adjustment, ASQ score remained significantly associated with $n-6 / n-3$ ratio $(\beta=-1.16$; $S E=0.37 ; P=0.0015)$. Association with total $n-3$ PUFAs tended to persist $(\beta=1834$; $S E=985$; $P=0.063$ )

Conclusions After adjustment for confounders, especially maternal education, higher $n$-3 PUFAs intake and thus lower $n-6 / n-3$ ratio in pregnancy food consumption were associated with better cognitive development in early childhood. We observed similar results with prepregnancy lipids intake. Our study suggests a role of prenatal nutrition on childhood cognitive development.

\section{MANAGEMENT OF NEWBORNS WITH SUSPECTED OR PROVEN CONGENITAL TOXOPLASMA INFECTION IN THE FIRST TEN DAYS OF LIFE}

doi:10.1136/archdischild-2012-302724.0208

P Garcia-Meric. Université de la Mditerranée, Marseille, France
Congenital toxoplasmosis is caused by transplacental fetal contamination following maternal primary infection.

Risk of transmission increases with gestational age. Severity is higher when transmission occurs before 20 WG leading to abortion, fetal loss, prematurity or severe fetal damage (particulary neurological). After this period, infection mainly affects the eye.

Since establishment of prenatal screening in Austria and France, toxoplasmosis has declined. Early maternal treatment (spiramycin or pyrimethamine-sulfonamide) has shown a lower incidence of fetal sequelae. Combination of ultrasound follow-up, fetal MRI, real time PCR on amniotic fluid allows antenatal diagnosis of severe forms of CT in which termination of pregnancy are accepted. Only $15 \%$ of infected liveborn children have clinical signs.

In countries with high incidence of CT and without national program, prenatal and/or neonatal screening are required to improve medical care and minimize sequelae (chorioretinitis, hydrocephalus ...). Low prevalence countries recommand neonatal screening or prevention rules.

In case of prenatal diagnosis, clinical examination, serological tests (detection of specific IgM/IgA confirmed at day 10, comparaison of immunologic mother- child profiles), transfontanellar ultrasonography and ocular fundus are performed at birth. In lack of prenatal screening, when children are symptomatic, maternal serology (with avidity test), PCR on placenta, neonatal lumbar punction and tomography are added. In asymptomatic children, diagnosis will be evocated when complications appear (visual impairment, psychomotor delay, seizures...).

Serological tests should be interpreted cautionnely in early or late maternal infection, maternal treatment without amniocentesis. Thus infected children have to be treated early to reduce risk of chorioretinitis.

\section{MANAGEMENT OF CHILDREN WITH SUSPECTED OR PROVEN CONGENITAL TOXOPLASMOSIS FROM DAY 10 TO THE END OF THEIR FIRST YEAR}

doi:10.1136/archdischild-2012-302724.0209

M Wallon. Service de Parasitologie, Hopital de la Croix-Rousse, Hospices Civils de Lyon, Lyon, France

Management from Day 10 to Day 365 has two goals according to the results of the work-up performed at birth, and eventually in utero. The first is to start treatment and surveillance in infected newborns and, the second, applying to settings where prenatal screening is performed, is to confirm the absence of infection in newborns who are born from a mother who seroconverted during pregnancy but who show no signs of infection at birth.

When congenital infection is proven the standard attitude in France is to start treatment without delay even newborns with no clinical signs. Treatment relies on a combination of pyrimethamine and sulfonamides but there is no consensus on the type of sulfonamides, on the dosages and rhythm of administration and on the length of treatment, ranging from 3 to 24 months. Children under treatment should be monitored regularly for side effects. The decrease of IgG under treatment is a normal evolution and should not be interpreted as a sign of non-infection. Regular neurological and ophthalmological examinations in the first year of life are also important to detect any signs that would deserve special attention.

In the second case, the absence of clinical and biological signs in utero or at birth significantly decreases the probability of infection. Repeated serological tests remain however necessary to fully exclude infection by monitoring the decrease of $\operatorname{IgG}$ to undetectable levels. Any neosynthesis of IgG would indicate that the child is infected and warrant starting the same treatment as in infected infants. 